\title{
Quantitative blush evaluator (QuBE) accurately quantifies microvascular dysfunction in patients with ST-Elevation Myocardial Infarction; comparison with Cardiovascular Magnetic Resonance
}

\author{
Christian Hamilton-Craig ${ }^{1 *}$, Italo Porto ${ }^{2}$, Giovanni Luigi De Maria², Luigi Natale², Filippo Crea²
}

From 2011 SCMR/Euro CMR Joint Scientific Sessions

Nice, France. 3-6 February 2011

\section{Introduction}

\section{Background}

After ST-elevation myocardial infarction (STEMI), microvascular obstruction (MVO) can be assessed using angiographic myocardial 'blush' grade (MBG), subject to interoperator variability. Quantitative blush evaluator (QuBE) is a free computer-calculated algorithm, with improved reproducibility. We aimed to compare QuBE in detecting $\mathrm{MVO}$ and its severity in comparison to the gold standard, cardiovascular magnetic resonance (CMR).

\section{Methods}

Fifty-two STEMI treated with successful primary-PCI were enrolled. QuBE values were blindly calculated on post-procedure angiogram and patients divided in tertiles (ter) according to QuBE values. All patients underwent CMR (GE 1.5T) 4-7 days after STEMI, for quantitative assessment of infarct size (IS), area-at-risk (AAR), myocardial salvage index (MSI), MVO (both as first pass MVO (FP-MVO) and delayed-enhancement MVO (DE-MVO)), and presence of intramyocardial hemorrhage on T2-weighted sequences. Indices were measured blindly by two expert readers with SMCR level 3, using ReportCard (v4.0) software. LGE infarct size was quantified using threshold $>5 \mathrm{SD}$, T2 sequence $>$ SD as previously described. MVO was manually

${ }^{1}$ University of Queensland, Brisbane, Australia

Full list of author information is available at the end of the article traced, and hemorrage identified as the dark band within bright signal on T2

\section{Results}

$\mathrm{QuBE}$ values were inversely related to IS, both as percentage (R: -0.4; p:0.001) and as mass (R:-0.4; p: 0.008), to DE-MVO, both as percentage $(\mathrm{R}:-0.7 ; \mathrm{p}<0.001)$ and as mass (R: $-0.7 ; \mathrm{p}<0.001$ ), to FP-MVO (R:-0.4; $\mathrm{p}$ : 0.002 ), and positively related to MSI (R: 0.4; p: 0.007). Moreover patients with intramyocardial hemorrhage had significantly lower $\mathrm{QuBE}$ values (3.9; 3.5-8.0 vs 12.2 ; 8.216.0; p:0.001). At receiver operating characteristic (ROC) curve analysis, QuBE accounted for an area under the curve of 0.88 (CI 95\% 0.7-0.9; p: 0.001) for both DEMVO and haemorrhage detection. Figure 1.

\section{Conclusions}

This is the first report to compare quantitative computer-based assessment of angiographic perfusion with comprehensive parameters of microvascular function, infarct size and salvage on early CMR in STEMI patients. We demonstrate the important finding that QuBE values correlate strongly with microvascular obstruction, Intramyocardial hemorrage and myocardial salvage by CMR. This validates the use of QuBE for assessment of tissue reperfusion in the clinical setting, and represents and important advancement in postprocedural evaluation of reperfusion in the cathlab. Our data suggest that a quantitative evaluation of myocardial blush after STEMI will accurately risk-stratify patients, and provide useful prognostic information, better 


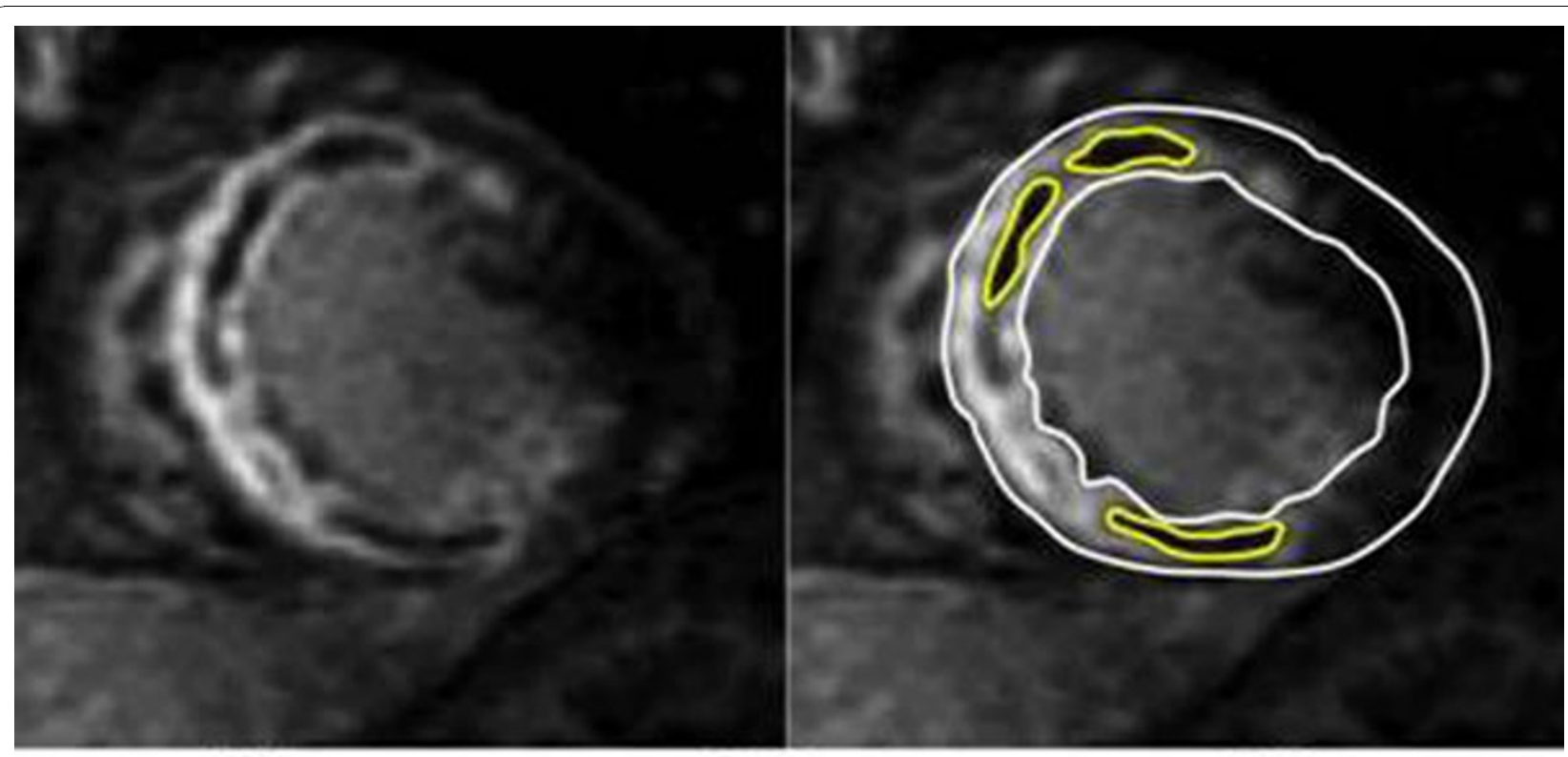

ROC Curve

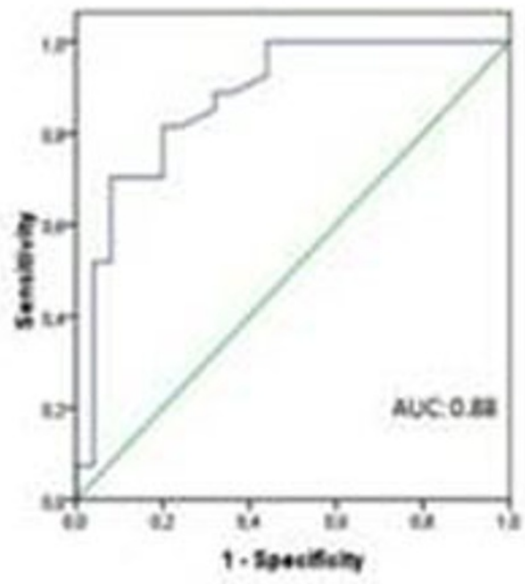

DE-MVO

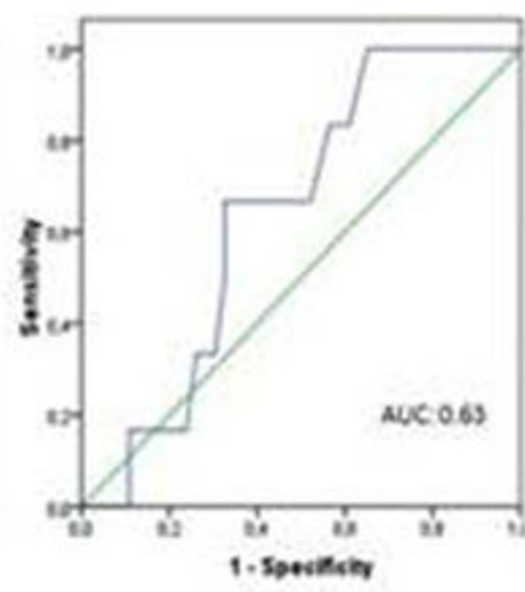

FP.MVO

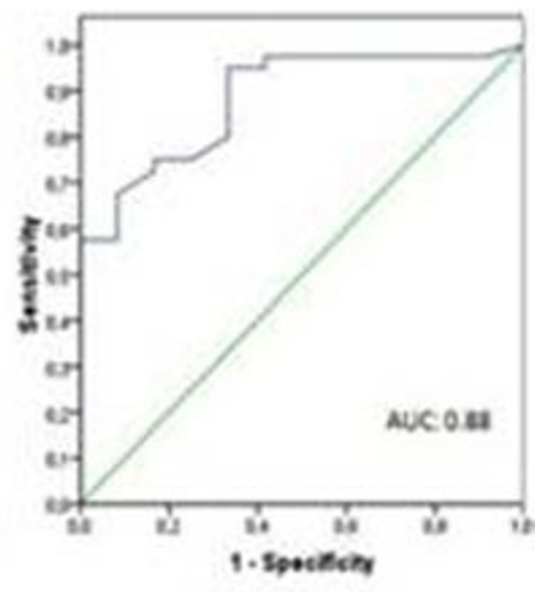

Hemorrhare

Figure 1 ROC curves for sensitivity and specificity of QuBE in detecting CMR derived values of DE-MVO, FP-MVO and hemorrhage.

informing decisions such as the selection of patients requiring further imaging (eg by CMR).

\section{Author details}

${ }^{1}$ University of Queensland, Brisbane, Australia. ${ }^{2}$ Catholic University of the Sacred Heart, Rome, Italy.

Published: 2 February 2011

doi:10.1186/1532-429X-13-S1-053

Cite this article as: Hamilton-Craig et al:: Quantitative blush evaluator (QuBE) accurately quantifies microvascular dysfunction in patients with ST-Elevation Myocardial Infarction; comparison with Cardiovascular Magnetic Resonance. Journal of Cardiovascular Magnetic Resonance 2011 13(Suppl 1):053.

\section{Submit your next manuscript to BioMed Central} and take full advantage of:

- Convenient online submission

- Thorough peer review

- No space constraints or color figure charges

- Immediate publication on acceptance

- Inclusion in PubMed, CAS, Scopus and Google Scholar

- Research which is freely available for redistribution 